\title{
Usefulness of Venous Pressure Measurement in Endovascular Treatment of Budd-Chiari Syndrome: A Retrospective Cohort Study
}

\author{
Ken Kageyama ${ }^{1}$, Akira Yamamoto ${ }^{1}$, Atsushi Jogo ${ }^{1}$, Takehito Nota ${ }^{1}$, Kazuki Murai ${ }^{1}$, \\ Satoyuki Ogawa ${ }^{1}$, Mariko M. Nakano ${ }^{1}$, Etsuji Sohgawa ${ }^{1}$, Shinichi Hamamoto ${ }^{1}$, \\ Masao Hamuro $^{2}$, Toshio Kaminou ${ }^{3}$, Norifumi Nishida ${ }^{4}$, Kanae Takahashi ${ }^{5}$, \\ Kouji Yamamoto ${ }^{5}$ and Yukio Miki ${ }^{1}$
}

\begin{abstract}
:
Objective Therapeutic predictors derived from the venous pressure before therapy have not been identified for Budd-Chiari syndrome (BCS). The aim of this study was to determine whether or not measuring the distal pressure or pressure gradient was useful for predicting treatment efficacy in BCS.

Methods We retrospectively analyzed seven consecutive patients diagnosed with symptomatic BCS at our hospital between 2008 and 2017. Distal and proximal venous pressures at occlusion sites of BCS were measured before treatment in all cases. The pressure gradient was defined as the difference between distal and proximal venous pressures. A receiver operating characteristics (ROC) analysis was performed for venous pressures.

Results Percutaneous old balloon angioplasty (POBA) was performed in seven cases, with technical success achieved in all cases $(100 \%)$. No complications were encountered. The median primary patency was 574 (interquartile range, 439.5-1,056.5) days. The 1-year primary patency rate was $71.73 \%$. Six cases $(85.7 \%)$ showed resolution of symptoms, representing clinical success. The ROC analysis revealed a high distal pressure (area under the ROC curve $=0.83$, cut-off $=12 \mathrm{mmHg}$ ) as a predictor of treatment efficacy of POBA for symptomatic BCS. In addition, the pressure gradient was considered significant from a clinical perspective, because the 6 successful cases with resolution of symptoms showed a large pressure gradient (range, 8-21 $\mathrm{mmHg}$ ) before treatment, whereas the failed case showed a relatively small pressure gradient $(7 \mathrm{mmHg})$.

Conclusion High distal pressure and a large pressure gradient might predict the treatment efficacy of balloon angioplasty for symptomatic BCS.
\end{abstract}

Key words: Budd-Chiari syndrome, venous pressure, POBA

(Intern Med 58: 2923-2929, 2019)

(DOI: 10.2169/internalmedicine.2704-19)

\section{Introduction}

Budd-Chiari syndrome (BCS) involves hepatic venous outflow obstruction at the suprahepatic inferior vena cava
(IVC) or in the hepatic veins, regardless of etiology (1). Hepatic venous outflow obstruction results in elevated hepatic sinusoidal pressure and hepatic congestion (2). Impediment of the hepatopetal portal venous flow leads to portal hypertension and diminished perfusion of the liver. The increase

\footnotetext{
${ }^{1}$ Department of Diagnostic and Interventional Radiology, Osaka City University Graduate School of Medicine, Japan, ${ }^{2}$ Department of Radiology, Izumiotsu Municipal Hospital, Japan, ${ }^{3}$ Department of Radiology, National Hospital Organization Osaka Minami Medical Center, Japan, ${ }^{4}$ Department of Radiology, Osaka Saiseikai Nakatsu Hospital, Japan and ${ }^{5}$ Department of Medical Statistics, Osaka City University Graduate School of Medicine, Japan

Received: January 21, 2019; Accepted: April 21, 2019; Advance Publication by J-STAGE: June 27, 2019

Correspondence to Dr. Ken Kageyama, kageyama@med.osaka-cu.ac.jp
} 
in hepatic sinusoidal pressure and portal hypertension leads to clinical hepatic impairment (3), manifesting as abdominal pain, hepatomegaly and splenomegaly, ascites, esophageal varices, portal hypertension, sinusoidal congestion with ischemic hepatocyte dysfunction, and even the development of fulminant hepatic failure (4).

BCS is managed by various treatment techniques, such as thrombolytic therapy, angioplasty with or without stenting, transjugular intrahepatic portosystemic shunts (TIPS), surgical shunt, and liver transplantation (5). However, controlled trials of therapies for BCS are lacking, and recommendations have been based on case series and expert opinions (3). Some investigations have measured the pressure distal and proximal to the occlusion site (far from and close to the heart, respectively, based on an occlusion site as a reference) before and after treatment (6-10). Percutaneous old balloon angioplasty (POBA), TIPS, and surgical shunt resulted in decreases in the distal pressure and the pressure gradient after treatment (6-10). The drop in these pressures is quite rational. However, to our knowledge, no reports have identified predictors for the treatment efficacy of POBA therapy by measuring pre-treatment pressures.

Even though patients are diagnosed with BCS based on radiological images and laboratory data, some have shown a low distal pressure and a small pressure gradient. Whether or not BCS patients showing a low distal pressure and a small pressure gradient should be treated remains unclear. We hypothesized that the distal pressure or the pressure gradient before treatment might be useful as a therapeutic predictor for the resolution of symptoms, representing clinical success, in POBA therapy.

The purpose of this study was to evaluate whether or not the measurement of the distal pressure or the pressure gradient is useful for predicting treatment efficacy in BCS.

\section{Materials and Methods}

\section{Patients}

This retrospective study was approved by the university committee on human investigation, and informed consent was obtained from all individual patients included in this study. We retrospectively analyzed seven consecutive patients diagnosed with symptomatic BCS at our hospital between May 2008 and April 2017.

\section{Diagnoses}

BCS was diagnosed from the medical history, clinical symptoms, findings from blood testing, and images from contrast-enhanced computed tomography (CT) that directly visualized a focal obstruction at the suprahepatic IVC or in the hepatic veins. X-ray venography also detected a focal obstruction. Cardiac, pericardial, and renal diseases were strictly excluded by cardiac and renal inspections. BCS has very similar symptoms to sinusoidal obstruction syndrome (SOS). SOS, which is associated with extensive obstruction of all hepatic veins, should be excluded from CT images and the medical history regarding chemotherapy, radiation therapy, or transplantation should be investigated in order to confirm BCS (11).

\section{Measurement of venous pressures}

The distal and proximal venous pressures at the occlusion site of BCS were measured before treatment in all cases. The pressure gradient was defined as the difference between the distal and proximal venous pressures. After POBA treatment, the distal and proximal pressures were also measured in the same manner as before treatment.

\section{Treatment}

Two out of three 4-Fr J-curved, straight or pig-tail catheters (RC-09, MEDIKIT, Tokyo, Japan; Impress STR or Pigtail, Merit Medical, South Jordan, USA) were inserted into the IVC distal to the occlusion site or into the right atrium proximal to the occlusion site via a transfemoral or transjugular approach, respectively. Pressure at both sites was measured using 4-Fr catheters. Mono or dual venography was performed using one or two catheters (Fig. 1a). For treatment, a 0.035-inch guidewire (Radifocus, Terumo, Tokyo, Japan) was first advanced to the occlusion site. If passage through the occlusion site was not possible due to severe stenosis, a needle (Rösch-Uchida Transjuglar Liver Access Kit; COOK, Bloomington, USA) was inserted in order to open an alternative occlusion site (Fig. 1b). When the puncture was successful, a 4-Fr catheter followed the needle and entered the other side. When the catheter entered the other side through the occlusion site, a small amount of contrast media was injected through the catheter to confirm that the catheter was in a vessel. A 0.035-inch stiff guidewire (Amplatz; COOK) was replaced with a 4-Fr catheter for POBA. A balloon catheter (Armada, Abbott, Abbot Park, USA; MAXI LD, Cordis, Fermont, USA) was inserted over the stiff wire via the jugular or femoral vein (Fig. 1c). The balloon was placed at the occlusion site, and the balloon was inflated manually three times for one minute (Fig. 1d, e). After dilation, venography was performed to confirm the expansion of the occluded vessels (Fig. 1f). After the procedure, heparin (10,000 units/day) was administered for 3 days, and then warfarin $(5 \mathrm{mg}$ /day) was administered orally to maintain an international normalized ratio of 2-3.

For cases with hepatic stenosis, the technique for POBA was as described above. Transhepatic puncture was initially performed under sonography guidance to insert a 5-Fr sheath (Supersheath; MEDIKIT), 0.035-inch guidewire, and a 4-Fr catheter. At the end of the procedure, the sheath was removed while embolizing the transhepatic track using gelatin sponge (Spongel; Astellas, Tokyo, Japan).

\section{Follow-up}

A clinical examination, including a laboratory examination, was performed at 1, 3, and 6 months after POBA and 


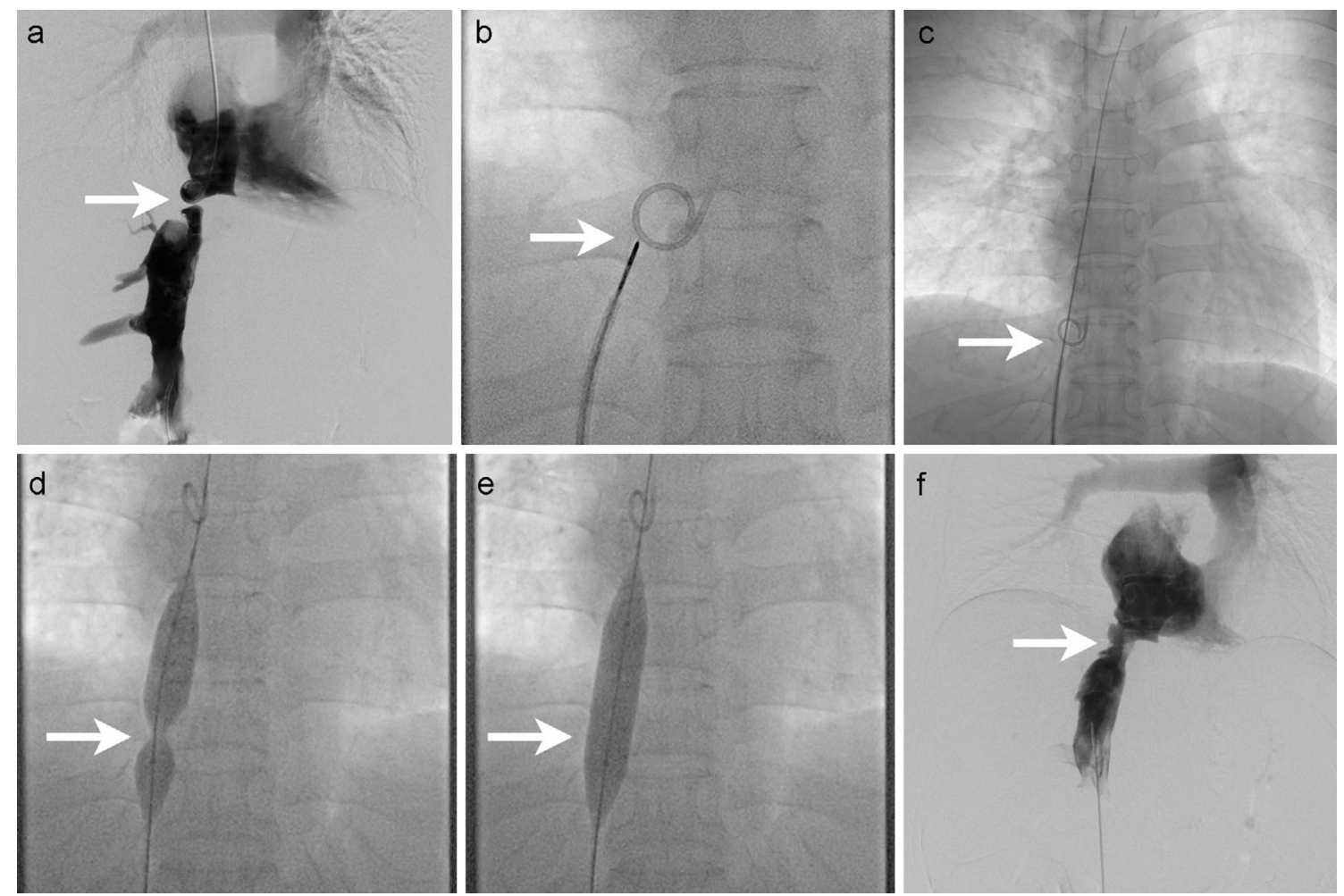

Figure 1. Procedure for POBA. a) Dual venography for anatomy control is performed. b) Needle puncture is performed from the distal side toward the catheter on the proximal side. c) After puncture, the guidewire penetrates the occlusion site. d) Expansion of the balloon catheter is started. e) Finally, the balloon achieves full expansion. f) After POBA, venography is performed from the distal side to confirm opening at the occlusion site. White arrows indicate an occlusion site (arrow). POBA: percutaneous old balloon angioplasty

once or twice a year as follow-up. CT was performed 3 or 6 months post-POBA. The Child-Pugh score was measured as the patient's liver status using data from the laboratory examination. Technical success was defined as restoration of the venous flow in the IVC and/or hepatic vein to the right atrium on venography and drops in both the distal pressure and the pressure gradient just after POBA. Primary patency was defined as the number of days between first POBA and the first appearance of occlusion as detected by CT. The primary patency rate was defined as the rate of patency at 1 year after POBA therapy. If reocclusion was suspected due to recurrence of clinical symptoms, CT was performed to confirm occlusion or stenosis. Re-POBA was performed for patients who experienced reocclusion or restenosis. Secondary patency was defined as the number of days between first POBA and the latest follow-up including re-POBA cases. The secondary patency rate was defined as the rate of patency at the latest follow-up, regardless of whether or not re-POBA had been performed.

Operative and post-operative complications were evaluated during follow-up. Clinical success was defined as resolution of symptoms, representing treatment efficacy in POBA therapy. The overall survival was evaluated as the period from treatment to the last clinical visit or confirmation of survival by phone. Data as above were analyzed at the time of discharge and after the last follow-up.

\section{Statistical analyses}

Patient characteristics were summarized as the median and interquartile range for continuous variables and frequency and percentage for categorical variables. The confidence interval (CI) for medians was calculated using the bootstrap method. Ten thousand sets of bootstrap samples were generated by resampling the original data, and the $95 \%$ $\mathrm{CI}$ for the median value was estimated. A receiver operating characteristic (ROC) curve analysis was performed in order to investigate the ability of the venous pressures to predict treatment efficacy, and the bootstrap optimism-corrected area under the ROC curve (AUC) of predictive performance was calculated for internal validation. When the ROC curve analysis showed statistically significant findings, the analysis also brought a cut-off value using the Youden index (12). Data were analyzed using the software programs JMP version 13.0.1 (SAS Institute, Cary, USA) and R version 3.3.4 (R Foundation for Statistical Computing, Vienna, Austria).

\section{Results}

\section{Patient characteristics}

Patient characteristics are summarized in Table. Five patients had IVC occlusion or stenosis, and the other two had 


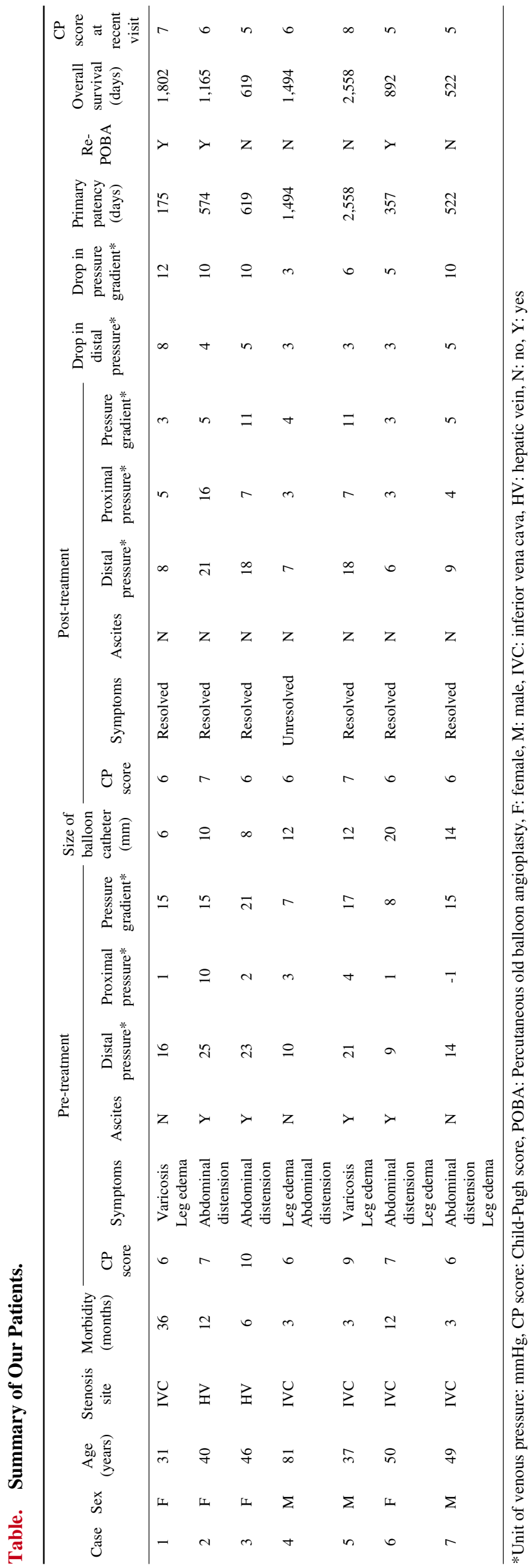

hepatic vein stenosis. The median distal and proximal pressures were 16 (interquartile range, 12-22) $\mathrm{mmHg}$ and 2 (13.5) $\mathrm{mmHg}$, respectively. The pressure gradient was 15 (11.5-16) $\mathrm{mmHg}$.

\section{Treatment}

POBA was technically successful in all 7 patients (100\%) (Fig. 2). Venography demonstrated significant improvement of IVC or hepatic venous outflow in all patients just after POBA. All seven patients showed a drop in the distal pressure and the pressure gradient after treatment. No operable complications were identified in any cases.

\section{Follow-up}

Primary patency was 574 (interquartile range, 439.5$1,056.5)$ days. The primary patency rate was $71.43 \%(5 / 7)$ within 1 year. Restenosis was observed in three patients. RePOBA treatment was performed for three patients after restenosis was detected by CT during the follow-up period. The secondary patency was $1,165(755.5-1,648)$ days. The secondary patency rate was $100 \%(7 / 7)$ at the latest followup. Six cases $(85.7 \%)$ showed resolution of symptoms immediately after treatment. Only one case did not show resolution of symptoms. The median follow-up for the seven patients was 1,165 (755.5-1,648) days. All seven patients were still alive as of the last follow-up. The seven patients who received POBA therapy showed just a 1-point difference in Child-Pugh score or no change from immediately after therapy to the latest follow-up.

\section{Pressure}

The venous pressures are summarized in Table. After treatment, the distal and proximal pressures were 9 (interquartile range, 7.5-18) $\mathrm{mmHg}(95 \% \mathrm{CI}, 7-18)$ and 5 (3.5-7) $\mathrm{mmHg}$ (95\% CI, 3-7), respectively. The pressure gradient was 5 (3.5-8) $\mathrm{mmHg}(95 \% \mathrm{CI}, 3-11)$. After treatment, the distal pressure tended to decrease, the proximal pressure tended to increase, and the pressure gradient tended to diminish. The drops in the distal pressure and pressure gradient were 4 (3-5) $\mathrm{mmHg}$ (95\% CI, 3-5) and 10 (5.5-10) $\mathrm{mmHg}$ (95\% CI, 5-10), respectively. The pre-treatment distal pressure in seven cases was identified as a predictor of treatment efficacy (representing clinical success) on the ROC analysis (AUC = 0.83) with $12 \mathrm{mmHg}$ as the cut-off (Fig. 3a and c). As the number of samples might not have been sufficient to make valid inferences from the AUC, we calculated the bootstrap optimism-corrected AUC for internal validation. That AUC was 0.85 , similar to the AUC of original data.

The pressure gradient in those cases was not identified as a predictor in the ROC analysis due to complete separation. The drops in the distal pressure and pressure gradient were also not identified in the ROC analysis due to complete or quasi-complete separation (Fig. 3b). Even though the pressure gradient was not a predictor according to the ROC analysis, the six successful cases showed a relatively large 


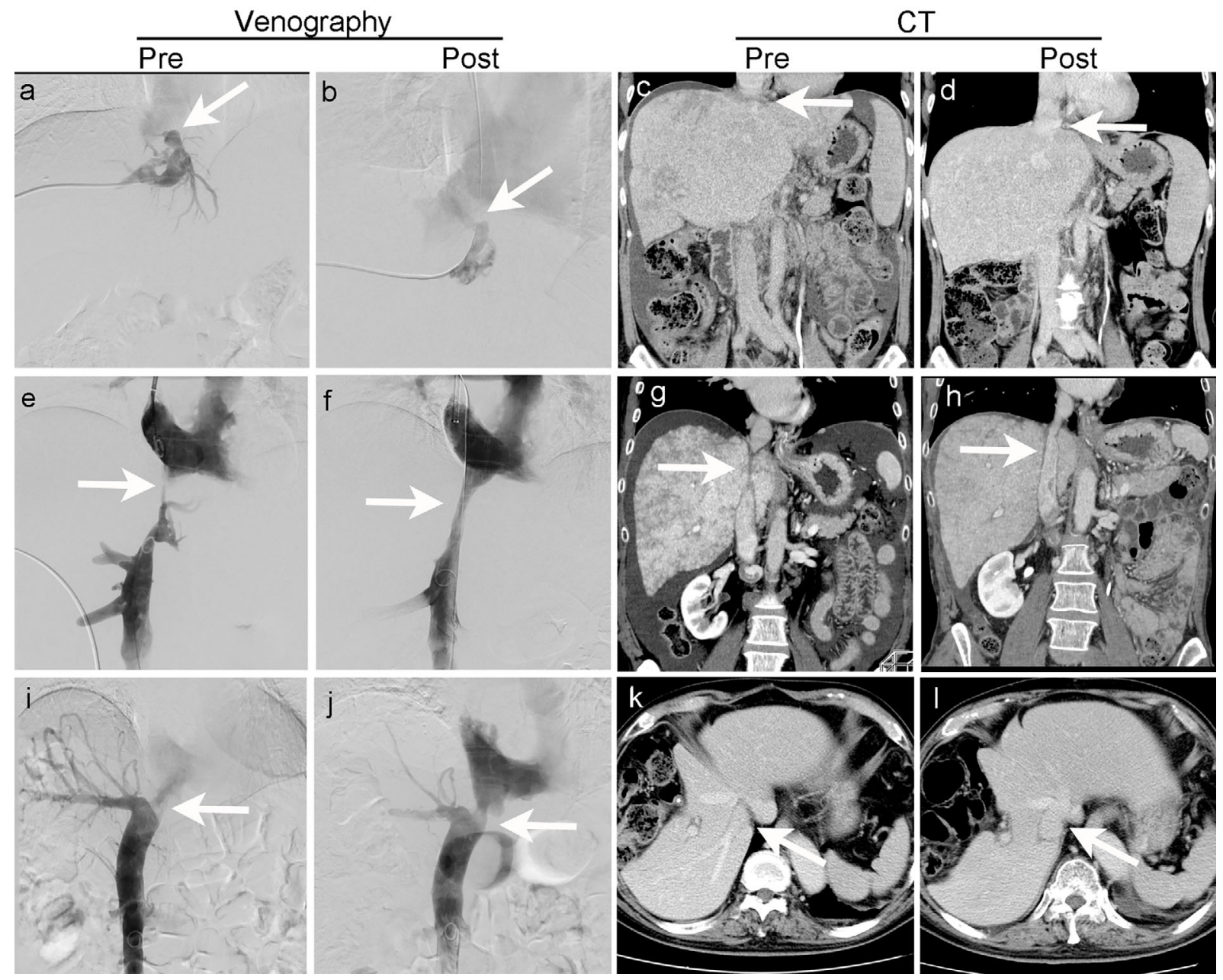

Figure 2. Three representative cases. Cases 3 and 6 showed improvement in clinical symptoms, whereas Case 4 showed no improvement in symptoms. From top to bottom, Case 3 is shown in the first row, Case 6 in the second row, and Case 4 in the third row. From left to right, pre-treatment venography is shown in the first column, post-treatment venography in the second column, pre-treatment CT in the third column, and post-treatment $\mathrm{CT}$ in the fourth column. All angiographic images were obtained via mono-venography except for Fig. 2e (dual-venography). White arrows indicate an occlusion or stenosis site (arrow).

pressure gradient (range, $8-21 \mathrm{mmHg}$ ) compared to the case of clinical failure $(7 \mathrm{mmHg})$. A high distal pressure and a large pressure gradient might predict the treatment efficacy of balloon angioplasty for symptomatic BCS.

\section{Discussion}

This is the first report to determine the indications for treatment using the distal pressure or the pressure gradient before therapy. Yang et al., Fisher et al., and $\mathrm{Fu}$ et al. showed that the average distal venous pressure at the occlusion site decreased after POBA treatment $(6,7,10)$. Similarly, Bachet et al. showed a drop in the pressure gradient after surgery (9). Previous studies only described decreases in the distal pressures and the pressure gradient after therapies. We attempted to establish grounds to indicate POBA treatment using venous pressure measurement.

Our study estimated that the cut-off value was $\geq 12 \mathrm{mmHg}$ for the distal pressure before therapy, based on ROC analysis. This cut-off value might be predictive of the efficacy of POBA treatment. Indeed, in the present study, 2 of the 7 cases had a low distal pressure below $12 \mathrm{mmHg}$, and one of them did not show any improvement in their clinical symptoms. The normal IVC pressure is reported to be 12.3 $\mathrm{mmHg}$ according to a previous report (13). If patients with BCS originally show a lower distal pressure, such as $<12$ $\mathrm{mmHg}$, the efficacy of POBA treatment for BCS is less likely, as the treatment is not able to achieve a substantial reduction in the distal pressure after treatment. Regarding the pressure gradient, no significant relationship was seen with an improvement of the clinical symptoms before therapy according to the ROC analysis. Although this study did not determine cut-off values for the pressure gradient before therapy, we speculate that a small pressure gradient does not lead to any marked improvement in clinical symptoms.

The natural history of BCS is not well known, as no co- 

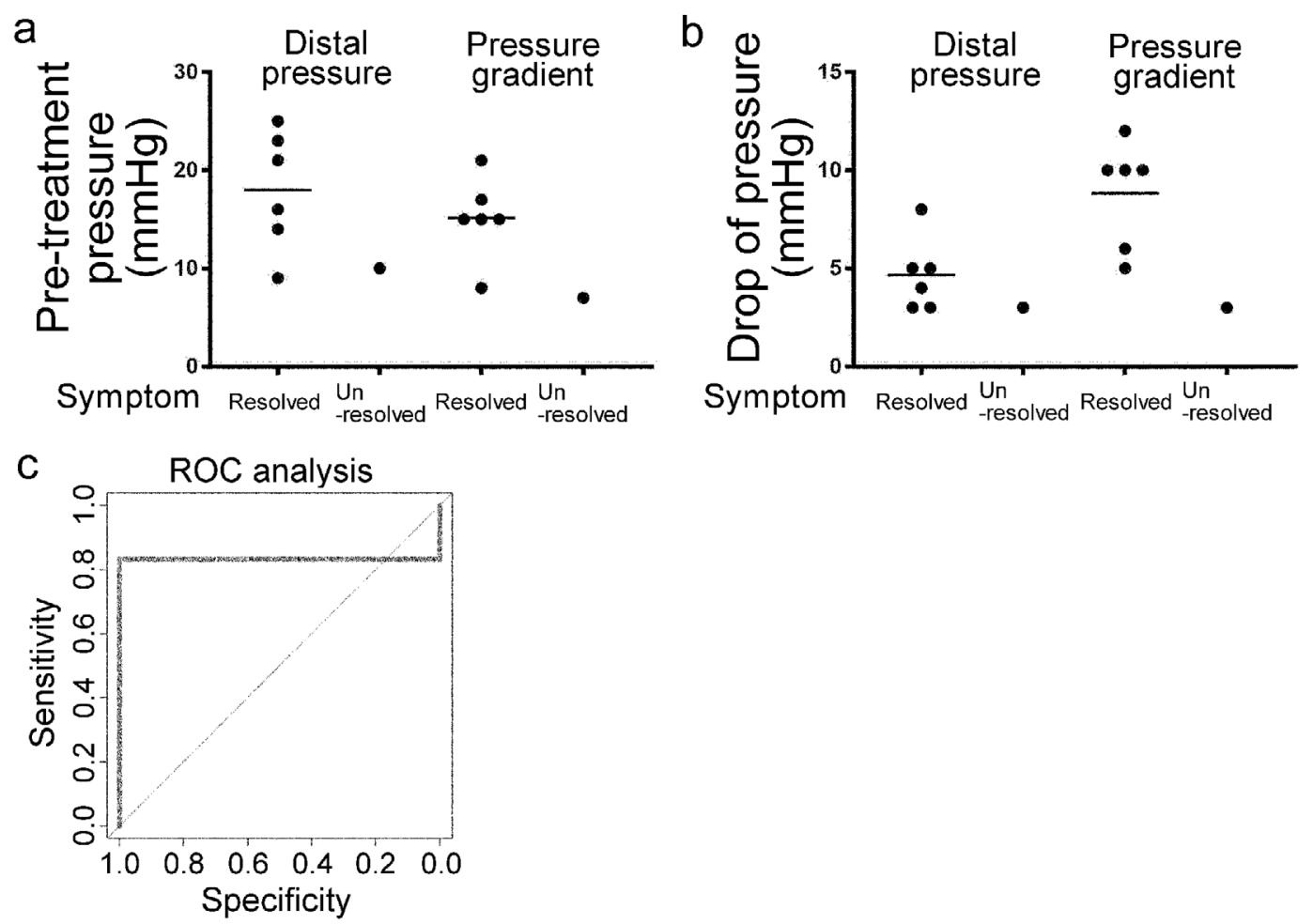

Figure 3. Chart of the measured pressures. a) Distal pressure and pressure gradient at pre-treatment. b) Drops in the distal pressure and pressure gradient after treatment. c) An ROC analysis of the distal pressure at pre-treatment.

hort studies have examined untreated patients $(11,14)$. Generally, BCS is thought to be a rare and potentially lifethreatening disease caused by obstruction of hepatic veins and/or IVC (14). However, in the most recently reported studies, overall 5-year survival rates over $90 \%$ have been achieved due to the increasing availability of therapeutic options $(8,15-18)$. Up to $20 \%$ of patients are completely asymptomatic, due to large collateral veins (19). Asymptomatic patients with collateral veins might show a low distal pressure or a small pressure gradient. In fact, the prognosis for asymptomatic patients is relatively good, even without interventional therapies such as POBA, TIPS, and surgical shunts (19). In cases with a high distal pressure or a large pressure gradient causing various symptoms in BCS patients, the reduction of these pressures might lead to improved clinical symptoms. Treatment of BCS is therefore useful for symptomatic patients with a high distal pressure, playing an important role in resolving current clinical symptoms as well as avoiding future liver failure. Venous pressure measurement may be critical for predicting the treatment efficacy before POBA.

Primary BCS is initially diagnosed at a median of 33 to 40 years old, according to previous reports (20-22), with a wide range of 16 to 83 years old. As the cause of primary BCS is unclear, we do not understand at what point BCS might develop. In the present study, the unresolved patient was 81 years old. Cardiac, renal, and liver failure were strictly excluded in order to diagnose BCS in this patient. His score of Child-Pugh classification was 6 points, indicat- ing moderate liver cirrhosis. However, the natural process of aging leads to hepatic functional and structural impairment (23). The liver state of this unresolved case might be irreversible even after POBA treatment due to liver cirrhosis.

POBA for BCS is a safe treatment with a low risk of complications. Since balloon dilatation was first described in 1974, the therapeutic pendulum has swung towards radiological intervention in patients with BCS (1). The patency rates with POBA were $97-97.3 \%$ at 5-8 years after therapy $(1,4)$. The survival of patients who received POBA was shown to be better than that in patients with surgical therapy (4). Endovascular management has resulted in lower rates of morbidity and mortality than open surgical techniques (2). Clinical symptoms and signs were significantly improved in all patients at discharge $(4,6)$. Although restenosis or obstruction following POBA has remained a major problem, re-POBA obtained secondary patency in the present study as well as in other studies.

Several limitations associated with the present study warrant mention. For example, this study included only a small number of cases. Therefore, in order to evaluate the statistical validity of this analysis, bootstrap validation was performed. Although the analysis was statistically valid, the small sample size reduced the trustworthiness. We must therefore recognize the results as being inferred in this study. Further investigations are still required in order to enhance the validity of the results. This report of venous pressure measurement in BCS seems to be most useful for answering scientific questions. BCS patients may need to be 
divided into two groups-IVC occlusion cases and hepatic vein occlusion cases-when we evaluate the distal pressure and pressure gradient. The hepatic vein pressure gradient was not measured in BCS patients in this study because the catheter could not reach the hepatic veins in some cases due to focal obstructions of the IVC or hepatic veins. This gradient is useful for evaluating portal hypertension, including BCS $(24,25)$.

In conclusion, a high distal pressure and large pressure gradient might be predictive of the treatment efficacy of balloon angioplasty for symptomatic BCS. However, in order to enhance the scientific validity, further investigations in larger populations will be required.

The authors state that they have no Conflict of Interest (COI).

\section{References}

1. Lee BB, Villavicencio L, Kim YW, et al. Primary Budd-Chiari syndrome: outcome of endovascular management for suprahepatic venous obstruction. J Vasc Surg 43: 101-108, 2006.

2. Copelan A, Remer EM, Sands M, Nghiem H, Kapoor B. Diagnosis and management of Budd Chiari syndrome: an update. Cardiovasc Intervent Radiol 38: 1-12, 2015.

3. Tripathi D, Macnicholas R, Kothari C, et al. Good clinical outcomes following transjugular intrahepatic portosystemic stentshunts in Budd-Chiari syndrome. Aliment Pharmacol Ther 39: 864-872, 2014

4. Ding PX, Zhang SJ, Li Z, Fu MT, Hua ZH, Zhang WG. Longterm safety and outcome of percutaneous transhepatic venous balloon angioplasty for Budd-Chiari syndrome. J Gastroenterol Hepatol 31: 222-228, 2016.

5. Menon KV, Shah V, Kamath PS. The Budd-Chiari syndrome. N Engl J Med 350: 578-585, 2004.

6. Yang XL, Cheng TO, Chen CR. Successful treatment by percutaneous balloon angioplasty of Budd-Chiari syndrome caused by membranous obstruction of inferior vena cava: 8-year follow-up study. J Am Coll Cardiol 28: 1720-1724, 1996.

7. Fisher NC, McCafferty I, Dolapci M, et al. Managing Budd-Chiari syndrome: a retrospective review of percutaneous hepatic vein angioplasty and surgical shunting. Gut 44: 568-574, 1999.

8. Rössle M, Olschewski M, Siegerstetter V, Berger E, Kurz K, Grandt D. The Budd-Chiari syndrome: outcome after treatment with the transjugular intrahepatic portosystemic shunt. Surgery 135: 394-403, 2004.

9. Bachet JB, Condat $\mathrm{B}$, Hagège $\mathrm{H}$, et al. Long-term portosystemic shunt patency as a determinant of outcome in Budd-Chiari syndrome. J Hepatol 46: 60-68, 2007.
10. Fu Y, Sun YL, Ma XX, et al. Necessity and indications of invasive treatment for Budd-Chiari syndrome. Hepatobiliary Pancreat Dis Int 10: 254-260, 2011.

11. DeLeve LD, Valla DC, Garcia-Tsao G. Vascular disorders of the liver. Hepatology 49: 1729-1764, 2009.

12. Obuchowski NA. ROC analysis. AJR Am J Roentgenol 184: 364372, 2005.

13. Hsia TY, Khambadkone S, Redington AN, de Leval MR. Effect of fenestration on the sub-diaphragmatic venous hemodynamics in the total-cavopulmonary connection. Eur J Cardiothorac Surg 19: 785-792, 2001.

14. Martens P, Nevens F. Budd-Chiari syndrome. United European Gastroenterol J 3: 489-500, 2015.

15. Darwish Murad S, Valla DC, de Groen PC, et al. Determinants of survival and the effect of portosystemic shunting in patients with Budd-Chiari syndrome. Hepatology 39: 500-508, 2004.

16. Plessier A, Sibert A, Consigny Y, et al. Aiming at minimal invasiveness as a therapeutic strategy for Budd-Chiari syndrome. Hepatology 44: 1308-1316, 2006.

17. Perelló A, García-Pagán JC, Gilabert R, et al. TIPS is a useful long-term derivative therapy for patients with Budd-Chiari syndrome uncontrolled by medical therapy. Hepatology 35: 132-139, 2002.

18. Eapen CE, Velissaris D, Heydtmann M, Gunson B, Olliff S, Elias E. Favourable medium term outcome following hepatic vein recanalisation and/or transjugular intrahepatic portosystemic shunt for Budd Chiari syndrome. Gut 55: 878-884, 2006.

19. Hadengue A, Poliquin M, Vilgrain V, et al. The changing scene of hepatic vein thrombosis: recognition of asymptomatic cases. Gastroenterology 106: 1042-1047, 1994.

20. Darwish Murad S, Plessier A, Hernandez-Guerra M, et al. Etiology, management, and outcome of the Budd-Chiari syndrome. Ann Intern Med 151: 167-175, 2009.

21. Uskudar O, Akdogan M, Sasmaz N, et al. Etiology and portal vein thrombosis in Budd-Chiari syndrome. World J Gastroenterol 14: 2858-2862, 2008.

22. Rajani R, Melin T, Björnsson E, et al. Budd-Chiari syndrome in Sweden: epidemiology, clinical characteristics and survival-an 18year experience. Liver Int 29: 253-259, 2009.

23. Sheedfar F, Di Biase S, Koonen D, et al. Liver diseases and aging: friends or foes? Aging Cell 12: 950-954, 2013.

24. Mueller S, Sandrin L. Liver stiffness: a novel parameter for the diagnosis of liver disease. Hepat Med 25: 49-67, 2010.

25. Hobolth L, Bendtsen F, Møller S. Indications for portal pressure measurement in chronic liver disease. Scand J Gastroenterol 47: $887-892,2012$

The Internal Medicine is an Open Access journal distributed under the Creative Commons Attribution-NonCommercial-NoDerivatives 4.0 International License. To view the details of this license, please visit (https://creativecommons.org/licenses/ by-nc-nd/4.0/).

\footnotetext{
(C) 2019 The Japanese Society of Internal Medicine
} Intern Med 58: 2923-2929, 2019 